\title{
A Case of Severe COVID-19 in a Patient with Good's Syndrome
}

\author{
Maria Duarte, Luciana Faria, Catarina Patronillo, Sónia da Costa Fernandes, Vera Seara \\ Internal Medicine Department, Centro Hospitalar Póvoa de Varzim - Vila do Conde, Portugal
}

Doi: 10.12890/2021_002976 - European Journal of Case Reports in Internal Medicine - @ EFIM 2021

Received: 05/10/2021

Accepted: $14 / 10 / 2021$

Published: $29 / 10 / 2021$

How to cite this article: Duarte M, Faria L, Patronillo C, da Costa Fernandes S, Seara V. A case of severe COVID-19 in a patient with Good's syndrome. EJCRIM 2021;8: doi:10.12890/2021_002976.

Conflicts of Interests: The authors declare there are no competing interests.

This article is licensed under a Commons Attribution Non-Commercial 4.0 License

\section{ABSTRACT}

Good's syndrome is a rare adult-onset combined immunodeficiency. The association of hypogammaglobulinaemia with a history of recurrent infectious or autoimmune manifestations in a middle-aged patient with evidence of a mediastinal mass should lead to the clinical suspicion of Good's syndrome. The mortality rate associated with infectious complications is high. Thus, although it is rare, the disease should be diagnosed early so that proper treatment can be started. Thymectomy and immunoglobulin replacement are the main therapeutic strategies. We describe the case of a patient with a history of thymoma and recurrent respiratory infections, with a late diagnosis of Good's syndrome in the context of severe organizing pneumonia secondary to COVID-19.

\section{LEARNING POINTS}

- Infection is the leading cause of mortality in patients with Good's syndrome.

- Early recognition of this rare disease and substitutive therapy with immunoglobulin may have an important impact on prognosis.

- Due to its rarity, data on Good's syndrome are scarce and its association with severe COVID-19 onset is still unclear.

- To the best of our knowledge, this is the fifth case report of COVID-19 in a patient with Good's syndrome, the third with a favourable outcome.

\section{KEYWORDS}

COVID-19, Good's syndrome, immunoglobulin

\section{CASE DESCRIPTION}

A 70-year-old woman presented to the emergency department (ED) with cough and dyspnoea beginning on that day, and fever and myalgia since the day before. Eleven days previously she had been diagnosed with COVID-19. On the eve and day of COVID-19 diagnosis, she presented headache, odynophagia and anosmia, and was later asymptomatic.

The patient had a history of dyslipidaemia, osteoporosis and immunoglobulin (Ig) G monoclonal gammopathy disease. Her surgical history included type A/B thymoma exeresis at 53 years of age in another hospital. Blood tests performed at that time revealed an lgM serum level of $41 \mathrm{mg} / \mathrm{dl}$ (normal range 55-300 mg/dl) with normal serum lgG (953 mg/dl) and lgA (171 mg/dl). She had no anaemia or hypercalcaemia, serum creatinine and beta-2 microglobulin levels were normal, and blood protein electrophoresis was unremarkable. At 60 years of age, she was referred to pulmonology consultation for recurrent respiratory infections. Computed tomography (CT) of the chest revealed bronchiectasis.

On examination in the $\mathrm{ED}$, her temperature was $38^{\circ} \mathrm{C}$, blood pressure $117 / 67 \mathrm{mmHg}$, and peripheral oxygen saturation was $97 \%\left(\mathrm{FiO}{ }_{2} 31 \%\right.$ ). Auscultation of the lungs revealed decreased breath sounds in the pulmonary bases, with bibasilar crackles. Arterial blood gas analysis confirmed hypoxaemia, with a $\mathrm{PaO}_{2} / \mathrm{FiO}_{2}$ ratio of 313 . On laboratory testing, the total white cell count was $5.17 \times 10^{\circ} / \mathrm{l}$, with lymphopaenia $\left(0.45 \times 10^{\circ} / \mathrm{l}\right)$, and the C-reactive protein level was $34.2 \mathrm{mg} / \mathrm{dl}$. The polymerase chain reaction test was positive for SARS-CoV-2. 
CT of the chest showed peripheral ground-glass opacities, with a tendency to confluence, which extended to the peribronchovascular region of the left upper lobe and right base. The patient was hospitalized and, due to worsening respiratory dysfunction, was transferred on the fifth day to an intensive care unit in another hospital. Severe organizing pneumonia secondary to COVID-19 was diagnosed. The patient was treated with high-flow nasal oxygen therapy, prone positioning, dexamethasone (12 mg daily for 10 days and subsequent slow weaning) and remdesivir (5 days), with progressive improvement. She returned to our hospital and was discharged after a total of 25 days of hospitalization.

Over 6 months of follow-up with internal medicine consultation, the patient had no new infectious events. Transthoracic echocardiography showed mild pulmonary hypertension. Pulmonary function tests, including spirometry, lung volumes, and diffusing capacity for carbon monoxide, were unremarkable. CT of the chest revealed total regression of the ground-glass opacities. Blood tests showed normal serum levels of haemoglobin, creatinine and calcium. The lgM serum level was $18 \mathrm{mg} / \mathrm{dl}$ (normal range 40-230 mg/dl), lgA $68 \mathrm{mg} / \mathrm{dl}$ (normal range $70-400 \mathrm{mg} / \mathrm{dl}$ ) and lgG $757 \mathrm{mg} / \mathrm{dl}$ (normal range $700-1600 \mathrm{mg} / \mathrm{dl}$ ). IgG subclass levels were analysed, revealing lgG2 $112 \mathrm{mg} / \mathrm{dl}$ (normal range 150-640 mg/dl) and lgG4 $2.8 \mathrm{mg} / \mathrm{dl}$ (normal range 8-140 mg/dl) with normal lgG1 and lgG3 levels. Blood protein immunoelectrophoresis confirmed the presence of a discrete monoclonal precipitation band compatible with IgG kappa monoclonal gammopathy disease. Immunophenotypic lymphocyte analysis revealed a complete absence of B-lymphocytes. T-lymphocyte subpopulations were in the normal range (T-CD8+ 586 cells $/ \mathrm{mm}^{3}$ and T-CD4+ 771 cells $/ \mathrm{mm}^{3}$ ). Specific antibody responses to T-dependent protein antigens (tetanus toxoid) and T-independent antigens (anti-polysaccharide antigens) were evaluated: anti-pneumococcal antibody response was normal but anti-tetanus toxoid antibody response was impaired (IgG $<0.10 \mathrm{IU} / \mathrm{ml}$ ). Serology for human immunodeficiency virus was negative and autoimmunity screening was unremarkable.

The absence of B-lymphocytes and hypogammaglobulinaemia at the expense of IgM and IgG subclasses (IgG2 and IgG4), associated with the history of thymoma and recurrent respiratory infections, are compatible with the diagnosis of Good's syndrome (GS).

The patient was referred to specialized consultation in immunodeficiencies in a reference centre, for treatment with intravenous Ig.

\section{DISCUSSION}

GS is a rare adult-onset combined immunodeficiency characterized by thymoma and hypogammaglobulinaemia ${ }^{[1]}$. Most cases occur between the ages of 40 and 70 , with equal distribution between the sexes ${ }^{[2]}$.

Although it was first described in 1954, its pathogenesis remains unclear. Immunological disturbances may include low to absent circulating B-lymphocytes, hypogammaglobulinaemia, decreased T-lymphocytes, inversion of the CD4+/CD8+ ratio, and functional defects in cellmediated immunity ${ }^{[2,3]}$.

The phenotype is variable. Infectious manifestations are more frequently due to encapsulated bacteria, fungi and viruses, predominantly affecting the respiratory and/or gastrointestinal tract, and may result in fatal outcomes. Autoimmune manifestations occur in up to $50 \%$ of GS patients, usually requiring intensive immunosuppressive therapy ${ }^{[2,4]}$.

Only $6-10 \%$ of patients with thymoma develop GS and no specific histopathological subtype has been associated with the syndrome ${ }^{[2]}$. As in the case we report, hypogammaglobulinaemia does not always improve after thymectomy, indicating thymoma management alone is not sufficient to resolve this disorder ${ }^{[1]}$. Other therapeutic strategies are mainly supportive with antimicrobials and Ig replacement, recommended in GS patients who present with recurrent infections ${ }^{[2]}$.

Infection remains the leading cause of mortality in patients with $\mathrm{GS}^{[1,2]}$ and prognosis is believed to be worse compared with other adult immunodeficiencies ${ }^{[1,3-5]}$. Moreover, compared with other primary humoral deficiencies, bronchiectasis caused by thymoma-associated hypogammaglobulinaemia has a higher mortality rate ${ }^{[5]}$. Humoral immunodeficiency (peripheral B-cell decrease in particular) has been correlated with a predisposition to severe infections ${ }^{[2]}$.

Improved recognition of this rare disorder, more frequent use of Ig replacement, expanded availability of various antimicrobial agents, and better supportive care in the recent decade, seem to be the main reasons for the downward trend in mortality ${ }^{[1]}$.

Due to its rarity and the absence of clear clinical and laboratory criteria, the diagnosis, as happened in this case, is often late, which may lead to a worse prognosis. Thus, the authors emphasize the importance of performing immunological screening for all patients with thymoma, as well as its regular repetition given the existing data on late development of immunodeficiency ${ }^{[2]}$.

As illustrated with this clinical case, patients with GS may also develop haematological malignancies, thus reinforcing the importance of follow-up.

This case had the particularity that the patient developed COVID-19, a disease also not yet fully clarified due to its recent appearance ${ }^{[6-8]}$. The patient was treated according to the best available evidence, with progressive improvement until clinical discharge. Being the only therapeutic strategy associated with a reduction in the infection rate and need for hospitalization in patients with $\mathrm{GS}^{[2,3]}$, Ig replacement therapy may be fundamental for the prevention and control of infectious manifestations in these patients. 


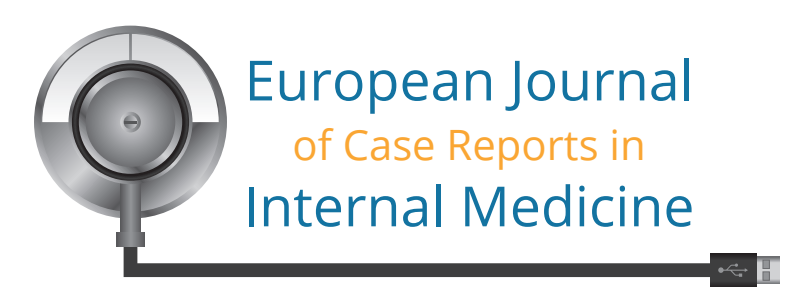

\section{REFERENCES}

1. Shi Y, Wang C. When the Good syndrome goes bad: a systematic literature review. Front Immunol 2021;12:679556. doi: 10.3389/fimmu.2021.679556.

2. Guevara-Hoyer K, Fuentes-Antrás J, Calatayud Gastardi J, Sánchez-Ramón S. Immunodeficiency and thymoma in Good syndrome: two sides of the same coin. Immunol Lett 2021;231:11-17. doi: 10.1016/j.imlet.2020.12.010.

3. Tamburello A, Castelnovo L, Faggioli P, Bompane D, Brando B, Gatti A, et al. Good's syndrome, a rare form of acquired immunodeficiency associated with thymomas. Clin Pract 2019;9(2):1112. doi: 10.4081/cp.2019.1112.

4. Jansen A, van Deuren M, Miller J, Litzman J, de Gracia J, Sáenz-Cuesta M, et al; Good syndrome Study Group. Prognosis of Good syndrome: mortality and morbidity of thymoma associated immunodeficiency in perspective. Clin Immunol 2016;171:12-17. doi: 10.1016/j.clim.2016.07.025.

5. Pu C, Sukhal S, Fakhran S. Humoral immunity in bronchiectasis: finding Good's syndrome. Case Rep Pulmonol 2015;2015:531731. doi: 10.1155/2015/531731.

6. London J, Boutboul D, Lacombe K, Pirenne F, Heym B, Zeller V, et al. Severe COVID-19 in patients with B cell alymphocytosis and response to convalescent plasma therapy. J Clin Immunol 2021;41(2):356-361. doi: 10.1007/s10875-020-00904-5.

7. López AL, Torre MG, Paolini MV, Juri MC, Fernández Romero DS. COVID-19 in adults with antibody deficiencies. Medicina (B Aires) $2021 ; 81$ (3):478-481.

8. Cos Esquius ML, López Montesinos I, Gimeno Martinez R, Eguía Núñez J, Caballero-Rabasco MA, Sánchez González B, et al. Severe COVID-19 pneumonia in Good syndrome with a favorable outcome. Clin Immunol 2021 Jun 29:108789. doi: 10.1016/j.clim.2021.108789. 